TRANSACTIONS OF THE

AMERICAN MATHEMATICAL SOCIETY

Volume 356, Number 9, Pages 3477-3482

S 0002-9947(03)03483-4

Article electronically published on November 25, 2003

\title{
THE BEST CONSTANT OF THE MOSER-TRUDINGER INEQUALITY ON $\mathrm{S}^{2}$
}

\author{
YUJI SANO
}

\begin{abstract}
We consider the best constant of the Moser-Trudinger inequality on $\mathbf{S}^{2}$ under a certain orthogonality condition. Applying Moser's calculation, we construct a counterexample to the sharper inequality with the condition.
\end{abstract}

\section{INTRODUCTION}

Let $(M, g)$ be a compact smooth Riemannian manifold of dimension $n$. We denote by $\mathbf{H}_{1}^{n}(M)$ the Banach space obtained from the vector space of $C^{\infty}$ functions $\varphi$ such that $\|\nabla \varphi\|_{L^{n}}$ is bounded, by completion with the norm

$$
\|\varphi\|_{\mathbf{H}_{1}^{n}}:=\sum_{l=0}^{1}\left\|\nabla^{l} \varphi\right\|_{L^{n}} .
$$

It is well known as an exceptional case of the Sobolev imbedding theorem that there exist constants $C, \mu$ and $\nu$ such that all $\varphi \in \mathbf{H}_{1}^{n}(M)$ satisfy

$$
\int_{M} e^{\varphi} d V \leq C \exp \left(\mu\|\nabla \varphi\|_{L^{n}}^{n}+\nu\|\varphi\|_{L^{n}}^{n}\right)
$$

(Trudinger, Aubin; cf. [2], p. 63). For applications, the best constant $\mu$ in (1.1) is essential. For example, in the case of the sphere, the following result was proved by Moser [5] when $n=2$, and Aubin [1] for general $n$. All $\varphi \in \mathbf{H}_{1}^{n}\left(\mathbf{S}^{n}\right)$ with integral equal to zero satisfy

$$
\int_{\mathbf{S}^{n}} e^{\varphi} d V \leq C \exp \left(\mu_{n}\|\nabla \varphi\|_{L^{n}}^{n}\right),
$$

where $C$ depends only on $n$, and

$$
\mu_{n}=(n-1)^{n-1} n^{1-2 n} \omega_{n-1}^{-1} .
$$

Here $\omega_{n}$ denotes the volume of $\mathbf{S}^{n}(1):=\left\{(x, y, z) \in \mathbf{R}^{3} \mid x^{2}+y^{2}+z^{2}=1\right\}$. Note that $\nu$ in (1.1) equals zero in this case. In particular, when $n=2$, (1.2) becomes

$$
\log \int_{\mathbf{S}^{2}} e^{\varphi} d \mu \leq \frac{1}{16 \pi} \int_{\mathbf{S}^{2}}|\operatorname{grad} \varphi|^{2} d \mu+\frac{1}{4 \pi} \int_{\mathbf{S}^{2}} \varphi d \mu+C,
$$

where $d \mu$ denotes the volume element of the canonical metric on $\mathbf{S}^{2}$. It is the best constant of the coefficient of $\int_{\mathbf{S}^{2}}|\operatorname{grad} \varphi|^{2} d \mu$ which is useful for Nirenberg's

Received by the editors March 12, 2003.

2000 Mathematics Subject Classification. Primary 34A26; Secondary 53C55.

Key words and phrases. The Moser-Trudinger inequality.

This research was partially supported by the Ministry of Education, Science, Sports and Culture of Japan, Grant-in-Aid for JSPS Fellows, 03340, 2003. 
problem: Characterize all Gauss curvature functions $K$ of Riemannian metrics $\tilde{g}$ conformal to the canonical metric $g_{0}$, so that $\tilde{g}=e^{-\varphi} g_{0}$. This requires a solution of the following nonlinear elliptic partial differential equation for a given $K$ :

$$
1-\Delta_{g_{0}} \varphi=K e^{-\varphi},
$$

where $\Delta_{g_{0}}:=-\nabla^{i} \nabla_{i}$ denotes the real Laplacian with respect to $g_{0}$. For the variational method we define a functional $S(\varphi)$ by

$$
S(\varphi):=-\log \int_{\mathbf{S}^{2}} K e^{\varphi} d \mu+\frac{1}{16 \pi} \int_{\mathbf{S}^{2}}|\operatorname{grad} \varphi|^{2} d \mu+\frac{1}{4 \pi} \int_{\mathbf{S}^{2}} \varphi d \mu .
$$

The existence of a solution of (1.4) is reduced to finding a minimum of $S(\varphi)$. Clearly $S(\varphi)$ is finite, but it is impossible to find a convergent subsequence of a given minimizing sequence when the best constant $\mu$ equals $\frac{1}{16 \pi}$. So we consider a sharper version of the Moser-Trudinger inequality (1.3) under addtional constraints on $\varphi$. For example, Aubin [1] proved that if $\varphi \in \mathbf{H}_{1}^{n}\left(\mathbf{S}^{n}\right)$ satisfies

$$
\int_{\mathbf{S}^{n}} \xi e^{\varphi} d V=0, \int_{\mathbf{S}^{n}} \varphi d V=0
$$

for all $\xi \in \Lambda$, which denotes the first eigenspace of the Laplacian, then

$$
\int_{\mathbf{S}^{n}} e^{\varphi} d V \leq C(\epsilon) \exp \left(\left(\frac{\mu_{n}}{2}+\epsilon\right)\|\nabla \varphi\|_{L^{n}}^{n}\right)
$$

for any $\epsilon>0$ and some constant $C(\epsilon)$. Also when $n=2$, Moser [6] proved that we can lower the best constant of $\mu$ to $\frac{1}{32 \pi}$ for all $\varphi \in C^{\infty}\left(\mathbf{R P}^{2}\right)$, i.e., $\varphi(\xi)=\varphi(-\xi)$ for $|\xi|=1$. Note that under these circumstances the minimum of $S(\varphi)$ exists, but these are just only partial answers to Nirenberg's problem.

In the context of Kähler geometry, the equation (1.4) can be regarded as a 1dimensional complex Monge-Ampère equation, since $\mathbf{S}^{2}$ is a 1-dimensional Kähler manifold. On this problem, similar results hold as follows. Let $X$ be a compact $m$-dimensional Kähler manifold with the positive first Chern class and its Kähler form $\omega$ representing the first Chern class. We define

$$
P(X, \omega):=\left\{\varphi \in C^{\infty} \mid \omega+\sqrt{-1} \partial \bar{\partial} \varphi>0\right\},
$$

and a functional $F_{\omega}$ on $P(X, \omega)[3]$ by

$$
\begin{gathered}
F_{\omega}(\varphi):=J_{\omega}(\varphi)-\frac{1}{V} \int_{X} \varphi \omega^{m}-\log \left(\frac{1}{V} \int_{X} e^{h_{\omega}-\varphi} \omega^{m}\right), \\
\frac{1}{V} \int_{X} \sqrt{-1} \partial \varphi \wedge \bar{\partial} \varphi \wedge \sum_{k=0}^{m-1} \frac{m-k}{m+1} \omega^{m-1-k} \wedge(\omega+\sqrt{-1} \partial \bar{\partial} \varphi)^{k},
\end{gathered}
$$

where $V=\int_{X} \omega^{m}$, and $h_{\omega}$ is uniquely determined by

$$
\begin{gathered}
\operatorname{Ric}(\omega)-\omega=\sqrt{-1} \partial \bar{\partial} h_{\omega}, \\
\int_{X}\left(e^{h_{\omega}}-1\right) \omega^{m}=0 .
\end{gathered}
$$

(See 4 for the way how $F_{\omega}$ and Mabuchi's K-energy are related to the Futaki character.) Note that the functional $S(\varphi)$ is equivalent to $F_{\omega_{K E}}(-\varphi)$ by regarding $\mathbf{S}^{2}$ as $\mathbf{C P}^{1}$. It may be commonly known among experts in this problem that the 
inequality (1.3) can be shown from the viewpoint of the existence of the KählerEinstein metrics. Concretely, if $X$ admits a Kähler-Einstein metric $\omega_{K E}, F_{\omega_{K E}}(-\varphi)$ is bounded from below by some absolute constant for all $-\varphi \in P\left(X, \omega_{K E}\right)$. Namely

$$
\frac{1}{V} \int_{X} e^{\varphi} \omega_{K E}^{m} \leq C \exp \left(J_{\omega_{K E}}(-\varphi)+\frac{1}{V} \int_{X} \varphi \omega_{K E}^{m}\right) .
$$

When the complex dimension of $X$ equals one, so that $X=\mathbf{C P}^{1}=\mathbf{S}^{2}$, 1.6 is equivalent to (1.3). So (1.6) can be regarded as a fully nonlinear generalization of the Moser-Trudinger inequality. In [7, Tian proved the sharper form of (1.6): if $\left(X, \omega_{K E}\right)$ is a Kähler-Einstein manifold, there are positive constants $\delta, C$ such that

$$
F_{\omega_{K E}}(-\varphi) \geq \delta \frac{J_{\omega_{K E}}(-\varphi)}{\left(1+\operatorname{osc}_{X} \varphi\right)^{2 m+2 / 2 m+2+\beta}}-C
$$

for any $-\varphi \in P\left(X, \omega_{K E}\right)$ perpendicular to $\Lambda_{1}$ (i.e., $\int_{X} \xi \cdot(-\varphi) \omega_{K E}^{m}=0$ for any $\left.\xi \in \Lambda_{1}\right)$. Here $\beta=\frac{1}{4} e^{-m}$ and $\Lambda_{1}$ denotes the space of the eigenfunctions of the complex Laplacian $\Delta_{K E}$ with eigenvalue 1 with respect to $\omega_{K E}$ (if this space is not empty, it is equivalent to the first eigenspace). Note that $\delta$ and $C$ may depend on the dimension of $X$, the first eigenvalue of $\Delta_{K E}$ which is greater than one, and the Sobolev constant with respect to $\omega_{K E}$. Moreover, Tian posed the following conjecture (see [7, p. 20, conjecture 5.5): if $X$ is a Kähler-Einstein manifold, there are constants $\delta>0, C_{\delta}$, which may depend on $\delta$, such that

$$
F_{\omega_{K E}}(-\varphi) \geq \delta \cdot J_{\omega_{K E}}(-\varphi)-C_{\delta}
$$

namely

$$
\frac{1}{V} \int_{X} e^{\varphi} \omega_{K E}^{m} \leq C_{\delta} \exp \left((1-\delta) J_{\omega_{K E}}(-\varphi)+\frac{1}{V} \int_{X} \varphi \omega_{K E}^{m}\right)
$$

for any $-\varphi \in P\left(M, \omega_{K E}\right)$ perpendicular to $\Lambda_{1}$. When $X=\mathbf{S}^{2}$, (1.7) is equivalent to

$$
\log \int_{\mathbf{S}^{2}} e^{\varphi} d \mu \leq \frac{1}{16 \pi}(1-\delta) \int_{\mathbf{S}^{2}}|\operatorname{grad} \varphi|^{2} d \mu+\frac{1}{4 \pi} \int_{\mathbf{S}^{2}} \varphi d \mu+C_{\delta}
$$

In a word, Tian's conjecture sugguests that the perpendicularity to $\Lambda_{1}$ could make the best constant of the Moser-Trudinger inequality on $\mathbf{S}^{2}$ smaller than $\frac{1}{16 \pi}$. If this conjecture is true, it will give a new partial answer to Nirenberg's problem. Note that this perpendicularity is different from Aubin's one (1.5) and is essential. In fact, it is known (Ding [3]) that we cannot make $\delta$ in (1.7) larger than zero without the perpendicularity if $X$ admits a nonzero holomorphic vector field (i.e., $\Lambda_{1}$ is not empty).

The purpose of this paper is to construct a counterexample to (1.8) for $\delta>0$ even if $\varphi$ is perpendicular to $\Lambda$. Note that $\Lambda$ is equivalent to $\Lambda_{1}$. In other words, the perpendicularity to $\Lambda$ cannnot make the best constant of the Moser-Trudinger inequality smaller than $\frac{1}{16 \pi}$. Our counterexample is based on Moser's proof in [5].

Remark 1.1. Our example is not a counterexample to Tian's conjecture, because it does not satisfy the Kähler condition.

The author would like to thank A. Futaki for leading him to this problem and for valuable advices, S. Bando and Y. Nakagawa for checking his paper and G. Tian for valuable indications. 


\section{Construction of the Counterexample}

As mentioned, our example closely follows the calculations of [5]. It suffices to find a sequence of functions $\{\varphi\}$ on $\mathbf{S}^{2}$ such that for any $\beta>1$

$$
\log \int_{\mathbf{S}^{2}} e^{\varphi} d \mu-\frac{1}{16 \pi \beta} \int_{\mathbf{S}^{2}}|\operatorname{grad} \varphi|^{2} d \mu+\frac{1}{4 \pi} \int_{\mathbf{S}^{2}} \varphi d \mu
$$

tends to infinity and that $\varphi$ is perpendicular to $\Lambda$. Theorem 2 in 5 implies that if $\varphi$ is a smooth function defined on $\mathbf{S}^{2}$ satisfying

$$
\int_{\mathbf{S}^{2}}|\operatorname{grad} \varphi|^{2} d \mu \leq 1, \int_{\mathbf{S}^{2}} \varphi d \mu=0,
$$

then there exists an absolute constant $C$ such that

$$
\int_{\mathbf{S}^{2}} e^{4 \pi \varphi^{2}} d \mu \leq C
$$

First, we will construct a sequence of functions $\{\varphi\}$ such that

(i) $\int_{\mathbf{S}^{2}}|\operatorname{grad} \varphi|^{2} d \mu=1$,

(ii) $\int_{\mathbf{S}^{2}} \varphi d \mu=0$

(iii) $\varphi$ is perpendicular to $\Lambda$,

(iv) $\int_{\mathbf{S}^{2}} e^{4 \pi \beta \varphi^{2}} d \mu$ tends to infinity for any $\beta>1$.

We introduce longitude $\phi$ and latitude $\theta$ on $\mathbf{S}^{2}$ so that the canonical metric is given by

$$
d s^{2}=d \theta^{2}+\cos ^{2} \theta d \phi^{2},|\theta|<\frac{\pi}{2},
$$

and $\theta= \pm \pi / 2$ corresponds to the two poles. Let $\varphi(\theta)$ be a radially symmetric function, that is, independent of $\phi$. Moreover, introducing the variable $t$ and the functions $w(t), \rho(t)$ by

$$
\begin{aligned}
e^{t / 2} & :=\tan \left(\frac{\theta}{2}+\frac{\pi}{4}\right), \\
w(t) & :=(4 \pi)^{1 / 2} \varphi(\theta), \\
\rho(t) & :=\frac{1}{e^{t}+e^{-t}+2}
\end{aligned}
$$

it suffices to find a sequence of functions such that (see [5])

(i') $\quad \int_{-\infty}^{+\infty} \dot{w}^{2} d t=1$,
(ii') $\quad \int_{-\infty}^{+\infty} w \rho d t=0$,

(iii) $\varphi$ is perpendicular to $\Lambda$,

(iv') $\int_{-\infty}^{+\infty} e^{\beta w^{2}} \rho d t$ tends to infinity for any $\beta>1$.

Regarding $\mathbf{S}^{2}$ as $\left\{(x, y, z) \in \mathbf{R}^{3} \mid x^{2}+y^{2}+z^{2}=1\right\}$, the basis of the first eigenfunctions is given by the coordinate functions $x, y$ and $z$. Since $\varphi(\theta)$ is radially symmetric, we may assume that $\varphi(\theta)$ is perpendicular to both $x$ and $y$. Then, we 
have only to require $\varphi$ to be perpendicular to $z$. Since $z$ is the eigenfunction of eigenvalue 1 (we are considering the complex Laplacian),

$$
0=\int_{\mathbf{S}^{2}} \varphi \cdot z d \mu=\int_{\mathbf{S}^{2}} \varphi \cdot \Delta z d \mu=\int_{\mathbf{S}^{2}} \nabla^{i} \varphi \nabla_{i} z d \mu .
$$

Note also that the condition (iii) reduces to

$$
\text { (iii') } \int_{-\infty}^{+\infty} \dot{w}(t) \dot{z}(t) d t=0 .
$$

Here we regard $z$ as a function of $t$.

Now we define $w_{\sigma}(t)$ as follows $(\sigma>0)$ :

$$
w_{\sigma}(t)= \begin{cases}\varepsilon, & t<-\varepsilon, \\ -t, & -\varepsilon \leq t<0, \\ t \sqrt{\frac{1-\varepsilon}{\sigma}}, & 0 \leq t<\sigma, \\ \sqrt{(1-\varepsilon) \sigma}, & \sigma \leq t .\end{cases}
$$

$0<\varepsilon<1$ is determined by $\sigma$ as indicated below.

It is obvious that $w_{\sigma}(t)$ as above satisfies $\left(\mathrm{i}^{\prime}\right)$. To fulfil (iii'), $w_{\sigma}(t)$ has to satisfy

$$
\int_{-\varepsilon}^{0}-\dot{z}(t) d t+\int_{0}^{\sigma} \sqrt{\frac{1-\varepsilon}{\sigma}} \dot{z}(t) d t=0
$$

hence

$$
z(-\varepsilon)=\sqrt{\frac{1-\varepsilon}{\sigma}} z(\sigma) .
$$

Note that $\varepsilon \rightarrow 0(\sigma \rightarrow+\infty)$, since $0<\varepsilon<1$ and $z(\sigma)$ tends to 1 as $\sigma \rightarrow+\infty$. Let $A_{\sigma}$ and $\tilde{w}_{\sigma}(t)$ denote $\int_{-\infty}^{+\infty} w_{\sigma}(t) \cdot \rho(t) d t$ and $w_{\sigma}(t)-A_{\sigma}$. Therefore $\tilde{w}_{\sigma}(t)$ satisfies $\left(\mathrm{i}^{\prime}\right),\left(\mathrm{ii}^{\prime}\right),\left(\mathrm{iii}^{\prime}\right)$. Note that $A_{\sigma}$ tends to 0 as $\sigma \rightarrow+\infty$, since

$$
0 \leq A_{\sigma} \leq \varepsilon \int_{-\infty}^{0} \rho d t+\sqrt{\frac{1-\varepsilon}{\sigma}} \int_{0}^{\sigma} t e^{-t} d t+\sqrt{(1-\varepsilon) \sigma} \int_{\sigma}^{+\infty} e^{-t} d t .
$$

In what follows, we will prove that

$$
\int_{-\infty}^{+\infty} e^{\beta \tilde{w}_{\sigma}^{2}} \rho d t \rightarrow \infty
$$

as $\sigma \rightarrow+\infty$. For $\sigma>0$ we have

$$
\begin{aligned}
\int_{-\infty}^{+\infty} e^{\beta \tilde{w}_{\sigma}^{2}} \rho d t & \geq \frac{1}{4} \int_{\sigma}^{+\infty} \exp \left(\beta \tilde{w}_{\sigma}^{2}-t\right) d t \\
& =\frac{1}{4} \exp \left(\beta\left(\sqrt{(1-\varepsilon) \sigma}-A_{\sigma}\right)^{2}-\sigma\right) \\
& =\frac{1}{4} \exp \left(\sigma\left(\beta\left(\sqrt{1-\varepsilon}-\frac{A_{\sigma}}{\sqrt{\sigma}}\right)^{2}-1\right)\right) .
\end{aligned}
$$

Since $\beta>1$ and $A_{\sigma} \rightarrow 0$ as $\sigma \rightarrow+\infty$, there is a positive constant $c$ such that

$$
\beta\left(\sqrt{1-\varepsilon}-\frac{A_{\sigma}}{\sqrt{\sigma}}\right)^{2}-1>c
$$

for $\sigma$ large enough. Then $\int_{-\infty}^{+\infty} e^{\beta \tilde{w}_{\sigma}^{2}} \rho d t$ tends to infinity as $\sigma \rightarrow+\infty$. 
Next we will construct a counterexample by making use of $w_{\sigma}(t)$, or rather

$$
\varphi_{\sigma}:=(4 \pi)^{-1 / 2} \tilde{w}_{\sigma}(t)
$$

Let us fix $\beta>\beta^{\prime}>1$. Now applying $a_{\sigma} \varphi_{\sigma}$ to (2.1) where $a_{\sigma}$ is a positive constant depending only on $\sigma$, what we should show is that by taking $a_{\sigma}$ appropriately,

$$
\int_{\mathbf{S}^{2}} \exp \left(a_{\sigma} \varphi_{\sigma}-\frac{1}{16 \pi \beta} a_{\sigma}^{2}\right) d \mu \rightarrow \infty
$$

as $\sigma \rightarrow+\infty$. Let $M(\sigma)$ be the maximum of $\varphi_{\sigma}$. Now we choose $a_{\sigma}$ such that $\frac{a_{\sigma}}{8 \pi \beta^{\prime}}=M(\sigma)$ for given $\sigma$. Since

$$
4 \pi \beta^{\prime} \varphi_{\sigma}^{2}-a_{\sigma} \varphi_{\sigma}+\frac{1}{16 \pi \beta} a_{\sigma}^{2}=4 \pi \beta^{\prime}\left(\varphi_{\sigma}-\frac{1}{8 \pi \beta^{\prime}} a_{\sigma}\right)^{2}+\frac{a_{\sigma}^{2}}{16 \pi}\left(\frac{1}{\beta}-\frac{1}{\beta^{\prime}}\right),
$$

we deduce

$$
a_{\sigma} \varphi_{\sigma}-\frac{1}{16 \pi \beta} a_{\sigma}^{2} \geq 4 \pi \beta^{\prime} \varphi_{\sigma}^{2}
$$

on $\left\{p \in \mathbf{S}^{2} \mid \varphi_{\sigma}(p)=M(\sigma)\right\}$. From (2.4) we obtain

$$
\begin{aligned}
& \int_{\mathbf{S}^{2}} \exp \left(a_{\sigma} \varphi_{\sigma}-\frac{1}{16 \pi \beta} a_{\sigma}^{2}\right) d \mu \geq \int_{\left\{\varphi_{\sigma}=M(\sigma)\right\}} \exp \left(4 \pi \beta^{\prime} \varphi_{\sigma}^{2}\right) d \mu \\
& =\int_{\sigma}^{+\infty} \exp \left(\beta^{\prime} \tilde{w}_{\sigma}(t)^{2}\right) \rho(t) d t \geq \frac{1}{4} \int_{\sigma}^{+\infty} \exp \left(\beta^{\prime} \tilde{w}_{\sigma}(t)^{2}-t\right) d t .
\end{aligned}
$$

Since $\beta^{\prime}>1$, the right hand side of (2.5) tends to infinity as $\sigma \rightarrow+\infty$ by the same argument as in (2.2). So (2.3) is proved. Thus we conclude that $\left\{a_{\sigma} \varphi_{\sigma}\right\}$ are the counterexample we want. Consequently we find that the best constant of the MoserTrudinger inequality cannot be lowered only by requiring the perpendicularity to $\Lambda$.

\section{REFERENCES}

1. T. Aubin: Meilleures constantes dans le théorème d'inclusion de Sobolev et un théorème de Fredholm non linéaire pour la transformation conforme de la courbure scalaire. J. Funct. Anal. 32 (1979), no. 2, 148-174. MR 80i:58043

2. T. Aubin: Some nonlinear problems in Riemannian geometry. Springer-Verlag, Berlin, 1998. MR 99i:58001

3. W. Ding: Remarks on the existence problem of positive Kähler-Einstein metrics. Math. Ann. 282 (1988), no. 3, 463-471. MR 90a:58186

4. A. Futaki and Y.Nakagawa: Characters of automorphism groups associated with Kähler classes and functionals with cocycle conditions. Kodai Math. J. 24 (2001), no. 1, 1-14. MR 2002c:32038

5. J. Moser: A sharp form of an inequality by $N$. Trudinger. Indiana Univ. Math. J. 20 (1970/71), 1077-1092. MR 46:662

6. J. Moser: On a nonlinear problem in differential geometry. Dynamical systems (Proc. Sympos., Univ. Bahia, Salvador, 1971), pp. 273-280. Academic Press, New York, 1973. MR 49:4018

7. G. Tian: Kähler-Einstein metrics with positive scalar curvature. Invent. Math. 130 (1997), no. 1, 1-37. MR 99e:53065

Department of Mathematics, Tokyo Institute of Technology, Oh-okayama, MeguroKU, TOKYO 152-8551, JAPAN

E-mail address: ysano@math.titech.ac.jp 